\title{
Early Diagnosis of Spontaneous Spinal Epidural Hematoma with Echo-Planar Gradient-Echo T2*-Weighted MR Imaging
}

\author{
Koichi Iwatsuki ${ }^{*}$, Toshiki Yoshimine1, Yu-Ichiro Ohnishi', Koshi Ninomiya1, \\ Toshika Ohkawa ${ }^{1}$, Kousuke Iwaisako² \\ ${ }^{1}$ Department of Neurosurgery, Osaka University Medical School, Osaka, Japan \\ ${ }^{2}$ Department of Neurosurgery, Kyoritu Hospital, Hyogo, Japan \\ Email: ${ }^{*}$ kiwatsuki@nsurg.med.osaka-u.ac.jp
}

Received 20 February 2015; accepted 5 March 2015; published 10 March 2015

Copyright (C) 2015 by authors and Scientific Research Publishing Inc.

This work is licensed under the Creative Commons Attribution International License (CC BY). http://creativecommons.org/licenses/by/4.0/

c) (i) Open Access

\begin{abstract}
Spontaneous spinal epidural hematoma (SSEH) is a rare idiopathic condition that leads to the acute onset of neurological deficits, which can have catastrophic consequences if not recognized early. It is important to make an early precise diagnosis. Spinal epidural hematoma has been increasingly recognized since the advent of magnetic resonance imaging (MRI). However, T1- and T2-weighted gradient-echo sequences are relatively less sensitive to the magnetic susceptibility effects of hemorrhage. Echo-planar gradient-echo T2*-weighted MR imaging (T2* MRI) is sensitive to these magnetic susceptibility effects and is commonly used for the detection of hemorrhage. We reported that the case of a 76-year-old man who presented with tetra paresis had an early diagnosis of spontaneous spinal epidural hematoma early diagnosed by T2* MRI.
\end{abstract}

\section{Keywords}

Spinal Epidural Hematoma, T2* MRI, Diagnosis, Hemorrhage

\section{Introduction}

Spontaneous spinal epidural hematoma (SSEH) is a rare condition that requires early diagnosis and treatment to ensure complete recovery of function. This condition has been increasingly recognized because patients presenting with rapid progressive neurological deficits of spinal cord origin undergo early evaluation with MRI. The diagnosis of SSEH, however, remains debated in the presentation of a differential diagnosis with spinal cord

\footnotetext{
"Corresponding author.
}

How to cite this paper: Iwatsuki, K., Yoshimine, T., Ohnishi, Y.-I., Ninomiya, K., Ohkawa, T. and Iwaisako, K. (2015) Early Diagnosis of Spontaneous Spinal Epidural Hematoma with Echo-Planar Gradient-Echo T2*-Weighted MR Imaging. Neuroscience \& Medicine, 6, 20-23. http://dx.doi.org/10.4236/nm.2015.61004 
involvement. Early diagnosis of SSEH is important to aid the therapeutic strategy decision. We report a case of spontaneous cervical SSEH diagnosed with T2* MRI in a patient with natural recovery without a surgical intervention.

\section{Case Report}

A 76-year-old male presented with acute onset progressive tetra paresis for $24 \mathrm{~h}$. His symptoms started as severe neck pain associated with the radiation of pain along his upper limb and difficulty in neck movements. Within a few hours, he recognized progressive tetra paresis and difficulty in walking. The next day, the symptoms progressed to tetraparesis with an inability to walk and hesitancy of micturition. He was transported to our emergency unit. His medical history was unremarkable: no trauma, smoking, drinking, or drug use. He was neither on anticoagulation nor antiplatelet medication at the time of presentation. Upon neurological examination, the power in extremities was $2 / 5$ (medical research council grading) with urinary retention. There was bilateral hypotonia with absent reflexes and the Babinski sign was negative. Sensory examination showed absent vibration sensation below $\mathrm{C} 5$ level and an impairment of joint position sensation in both feet.

An urgent MRI of the cervical spine revealed a dorsal extradural lesion at the C2-Th3 level which was iso/ hyper intense to the cord on T1-weighted images (T1WI) (Figure 1(a)) and iso/hyper intense with a band of hypo intensity on T2-weighted images (Figure 1(b)). A T2* MRI was taken under the suspicion of hemorrhage. It disclosed hypo intensity spots within the hyper intensity mass and a clear hypo intense rim (Figure 1(c)). The lesion showed blooming displacing the spinal cord anteromedially. His initial laboratory workup failed to show any significant abnormalities and the coagulation profile was normal. Given the acute presentation and the MRI findings, in particular the T2* MRI, a diagnosis of spontaneous cervico-thoracic epidural hematoma causing cord compression was suspected.

After emergency hospitalization, he showed gradual natural recovery. He could stand and walk 3 days after hospitalization. His urination had normalized 2 days after hospitalization. An MRI taken after 5 days, which revealed that the lesion was thinner, a hyper intensity on T1WI, and mixed intensity on T2WI and T2* images (Figures 2(a)-(e)). MRI Imaging Studies MRI was performed with a $1.5 \mathrm{~T}$ superconducting unit (Magnetorom Vision, Siemens) with a standard head coil to obtain axial fast spin-echo T2-weighted images (repetition time $[\mathrm{TR}] /$ echo time $[\mathrm{TE}] /$ excitations, $3600 / 96 / 2$, slice thickness $=5 \mathrm{~mm}$, gap $=1 \mathrm{~mm}$ ). The imaging matrix and field of view were $224 \AA \sim 256$ and $23 \mathrm{~cm}$, respectively. Axial single-shot echo-planar gradient-echo T2-weighted imaging (TE/excitations $25 / 1$, flip angle $90^{\circ}$ ) was also obtained with a slice thickness and gap of $5 \mathrm{~mm}$ and 1 $\mathrm{mm}$, respectively. The imaging matrix and field of view were $128 \AA \sim 128$ and $23 \mathrm{~cm}$, respectively.

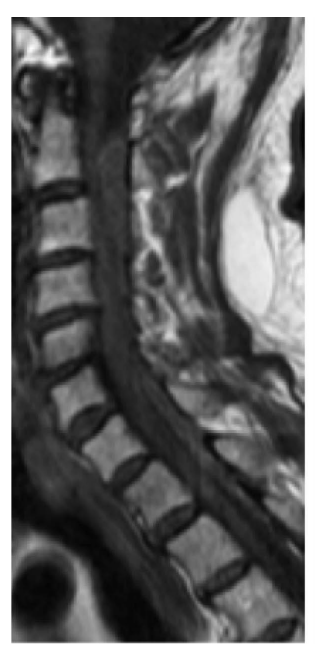

(a)

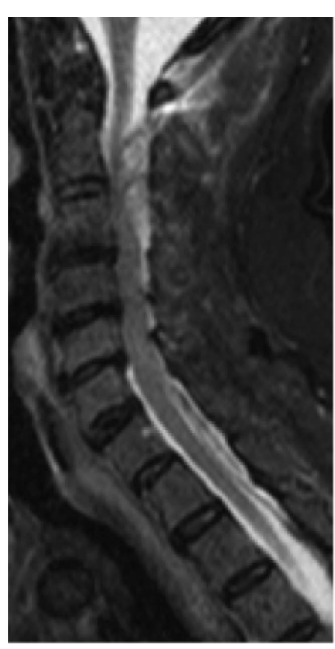

(b)

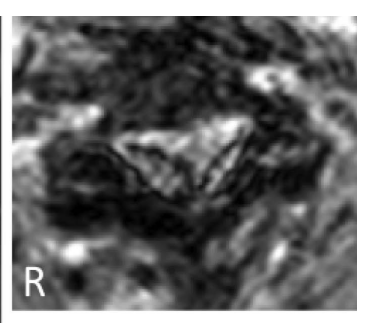

(c)

Figure 1. (a) A dorsal extradural lesion at the C2-Th3 level, which was iso/ hyperintense to the cord on T1-weighted images (b) iso/hyperintense with a band of hypointensity on T2-weighted images (c) hypointensity spots within a hyperintensity mass with a clear hypointense rim on T2* MRI. 


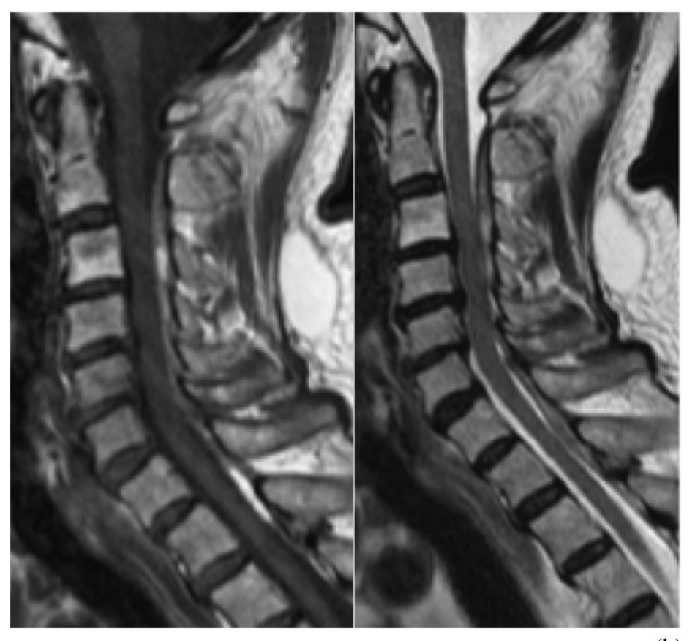

(a)

(b)

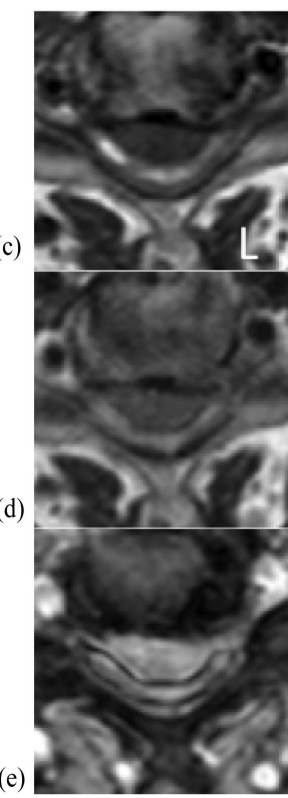

Figure 2. (a) (c) The lesion was becoming thin and revealed hyperintensity on T1WI and mixed intensity on T2WI (b) (d), T2* MRI (e).

\section{Discussion}

Spinal epidural hematoma was first described by Jackson in 1869 [1] and first treated surgically by Bain in 1897 [2]. The incidence of SSEH is estimated at $0.1 \cdot 100000^{-1} \cdot \mathrm{year}^{-1}$ [3]. Spinal epidural hematomas occur most frequently in the elderly but can occur at any age [4]. They are classified into two groups: nonspontaneous and spontaneous. Nonspontaneous epidural spinal hematomas may result from spinal taps, spinal anesthesia, trauma, pregnancy, bleeding diathesis, anticoagulant therapy, spinal hemangiomas, vascular malformations, hypertension, and neoplasms. Spinal epidural hematoma is capable of producing severe and irreversible neurologic deficits, and acute surgical intervension may be needed. The early precise diagnosis is crucial. Current literature supports both venous and arterialorigins as the source of spontaneous epidural hematomas [5]. The most widely accepted hypothesis for the source of bleeding is venous, because spinal epidural veins have no valves and are thus unprotected from changes in abdominal or thoracic pressure [5]. Increasing intrathoracic and intra-abdominal pressure leads to brief increases in intravenous pressure in valveless and thin-walled epidural veins, subsequently leading to their rupture. This accounts for cases that are reported occurring with activities such as straining, bending, coitus, coughing, and sneezing [6].

The investigation of choice is MRI. Following the inclusion of MRI into standard medical practice, the mean incidence of SSEH cases reported in the literature has increased further [7]. In the first $24 \mathrm{~h}$, an epidural hematoma is isotense to the cord on T1WI and is usually hyperintense, although it may be heterogeneous on T2WI. By $48 \mathrm{~h}$, the hematoma appears hyperintense on both T1WI and T2WI [3] [8]. The radiological differential diagnosis includes epidural abscess and spinal epidural lymphoma.

SSEH is acute onset and surgical management is recommended in the case of progressive symptoms, which can have catastrophic consequences if they are not recognized early in presentation. MRI is useful to verify diagnosis; however, T1WI and T2WI are relatively less sensitive to the magnetic susceptibility effects of hemorrhage. T2* MRI is sensitive to these magnetic susceptibility effects and is commonly used for the detection of hemorrhage [9]-[11]. T2* MRI requires a very short time for complete acquisition and is also sensitive to the effects of the local static magnetic field in homogeneities induced by the presence of hemosiderin [12]. Furthermore, T2* MRI can detect hyperacute hemorrhage because susceptibility is increased by the paramagnetic effect of deoxyhemoglobin, which is the earliest observable hemoglobin breakdown product on MRI [13]. Thus, T2* MRI is thought to be highly sensitive for detecting hemoglobin degradation products. In the case presented here, we could make a precise diagnosis of SSEH early on with T2* MRI and following up with it helped our decision on clinical course. T2* MRI might be recommended for evaluation of SSEH. 


\section{Conclusion}

Spinal epidural hematoma is capable of producing severe and irreversible neurologic deficits, and acute surgical intervention may be needed. The early precise diagnosis is crucial. We reported a case of spinal epidural hematoma diagnosed with T2* MRI early on and it was followed without a surgery. T2* MRI was useful for the early precise diagnosis of spinal epidural hematoma.

\section{Consent}

Written informed consent was obtained from the patient for publication of this case report and any accompanying images.

\section{References}

[1] Jackson, R. (1869) Case of Spinal Apoplexy. The Lancet, 94, 5-6. http://dx.doi.org/10.1016/S0140-6736(02)67624-X

[2] Bain, W.A. (1897) A Case of Haematorrachis. BMJ, 2, 455. http://dx.doi.org/10.1136/bmj.2.1912.455

[3] Holtas, S., Heiling, M. and Lonntoft, M. (1996) Spontaneous Spinal Epidural Hematoma: Findings at MR Imaging and Clinical Correlation. Radiology, 199, 409-413. http://dx.doi.org/10.1148/radiology.199.2.8668786

[4] Foo, D. and Roissier, A.B. (1981) Preoperative Neurological Status in Predicting Surgical Outcome of Spinal Epidural Hematomas. Surgical Neurology, 15, 389-401. http://dx.doi.org/10.1016/0090-3019(81)90178-6

[5] Awada, A., Russell, N., al Fayez, N., Naufal, R. and al Kohlani, H. (1998) Spontaneous Cervical Epidural Hematoma: Case Report. Spinal Cord, 36, 71-72. http://dx.doi.org/10.1038/sj.sc.3100521

[6] Liao, C.C., Hsieh, P.C., Lin, T.K., Lin, C.L., Lo, Y.L. and Lee, S.C. (2009) Surgical Treatment of Spontaneous Spinal Epidural Hematoma: A 5-Year Experience. Journal of Neurosurgery: Spine, 11, 480-486. http://dx.doi.org/10.3171/2009.4.SPINE08904

[7] Jamjoom, Z.A. (1996) Acute Spontaneous Spinal Epidural Hematoma: The Influence of Magnetic Resonance Imaging on Diagnosis and Treatment. Surgical Neurology, 46, 345-349. http://dx.doi.org/10.1016/S0090-3019(96)00149-8

[8] Avrahami, E., Tadmor, R., Ram, Z., Feibel, M. and Itzhak, Y. (1989) MR Demonstration of Spontaneous Acute Epidural Hematoma of the Thoracic Spine. Neuroradiology, 31, 89-92.

[9] Offenbacher, H., Fazekas, F., Schmidt, R., Koch, M., Fazekas, G. and Kapeller, P. (1996) MR of Cerebral Abnormalities Concomitant with Primary Intracerebral Hematomas. AJNR-American Journal of Neuroradiology, 17, 573-578.

[10] Greenberg, S.M., Finklestein, S.P. and Schaefer, P.W. (1996) Petechial Hemorrhages Accompanying Lobar Hemorrhage: Detection by Gradient-Echo MRI. Neurology, 46, 51-54. http://dx.doi.org/10.1212/WNL.46.6.1751

[11] Fazekas, F., Kleinert, R., Roob, G., Kleinert, G., Kapeller, P., Schmidt, R., et al. (1999) Histopathologic Analysis of Foci of Signal Loss on Gradient-Echo T2*-Weighted MR Images in Patients with Spontaneous Intracerebral Hemorrhage: Evidence of Microangiopathy-Related Microbleeds. AJNR-American Journal of Neuroradiology, 20, 637-642.

[12] Liang, L., Korogi, Y., Sugahara, T., Shigematsu, Y., Okuda, T., Ikushima, I., et al. (1999) Detection of Intracranial Hemorrhage with Susceptibility-Weighted MR Sequences. AJNR-American Journal of Neuroradiology, 20, 15271534.

[13] Patel, M.R., Edelman, R.R. and Warach, S. (1996) Detection of Hyperacute Primary Intraparenchymal Hemorrhage by Magnetic Resonance Imaging. Stroke, 27, 2321-2324. http://dx.doi.org/10.1161/01.STR.27.12.2321 